\title{
Forest Fire Detection Using Wireless Multimedia Sensor Networks and Image Compression
}

\author{
Fatima Bouakkaz $^{1 *}$, Wided Ali $^{2}$, Makhlouf Derdour ${ }^{2}$ \\ ${ }^{1}$ Computer Science Department, Superior National School of Computer Science (ESI), Oued Smar, Algiers 16000, Algeria \\ ${ }^{2}$ Computer Science Department, Tebessa University, Tebessa 12000, Algeria
}

Corresponding Author Email: f_bouakkez@esi.dz

https://doi.org/10.18280/i2m.200108

Received: 3 December 2020

Accepted: 15 February 2021

\section{Keywords:}

forest fire detection, images compression, wireless multimedia sensor network, Downsampling, grid chain protocol

\begin{abstract}
Recently, the issue of multimedia sensors received considerable critical attention, that led to the apparition of Wireless Multimedia Sensor Networks (WMSNs) WMSN that different from wireless sensor networks (WSN) by using multimedia sensors that can process video, audio, image data besides scalar data and send it to station base (SB). Multimedia data have a big volume bigger than scalar data and need more resources and consumed more energy. The ideal solution to solve the problems of WMSN (big volume, energy consumption) is data compression. Forest plays a critical role in our daily life we can summarize the importance of forests in human life. Among the most dangerous events the forest fires that happen because of natural or Man-made. Many methods used to detect forest fires the newest are: wireless multimedia sensor networks. Our system of detecting forest fire has been developed using a wireless multimedia senor network with two types of sensors (scalar, images). In the first phase when the scalar sensors detected a high temperature its announced alarm to activate the image sensors. In the second phase for detecting fire the image sensors, we used image processing tools. When the zone of fire in the image captured was detected the phase of compression started using the down sampling method. the final phase is transmission data to the station base using the grid chain transmission protocol technique, which allows a critical optimization of energy consumption. So, maximizing network life. The competence of the proposed system is achieved by minimizing size of image transmitted with grid chain routing protocol.
\end{abstract}

\section{INTRODUCTION}

A Wireless Multimedia Sensors Network is an extends of Wireless Sensor Network by adding multimedia sensors (images, video, or audio) where every node can sense, compress and send sensed images to the Station Base. Wireless multimedia sensor network WMSN has many applications the most known is multimedia surveillance and environmental monitoring using image processing techniques. Figure 1 shows a forest monitoring system using WMSN

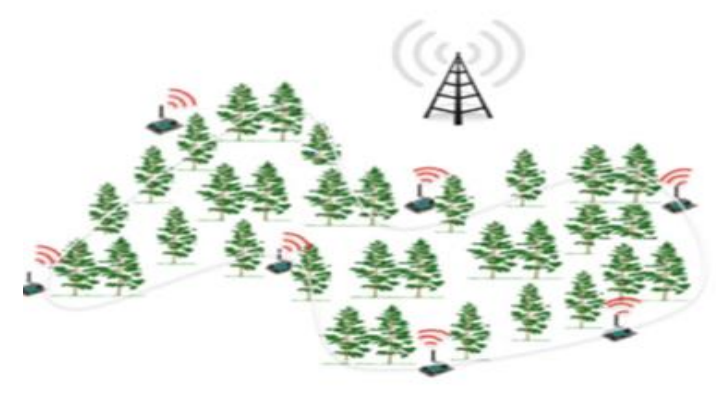

Figure 1. Forest monitoring system using WMSN

The big size of the data transmitted that consumed more energy of the network leads to be a serious issue of WMSN. According that, the key aim of this paper is that compression image that contain the fire with simple method in the multimedia senor with minimum consumption of energy to transmitted to the station base using grid chain protocol. Our protocol aims to achieve the following objectives:

a- summarizing the previous works of image compression Techniques and forest fire detection used in WMSN;

b-The proposed system is composed of two levels. The first level includes scalar sensors which can sense the temperature degree, the second level contain sensor nodes fitted with cameras which can sense images. The task of detection fire is to find fires in images and cutting the regions containing them and compress images and send it to the station base.

c- Minimizing size of image in each camera node led to reduce energy consumption in sensor that prolong network life.

$\mathrm{d}$-The use of grid chain routing protocol in the phase transmission also helped to minimize the energy consumption that maximizing the network life.

As mentioned in the abstract section, it will be rather easy to follow these rules as long as you just replace the "content" here without modifying the "form".

\section{IMAGE COMPRESSION TECHNIQUES USED IN WMSN}

The image formed of set pixels strongly correlated together and contained big redundancies which affected negatively the volume of storage and the transmission. Energy consumption in wireless sensor networks is the main problem, especially if the data transmitted are images, due to the big size of images compared by scalar data. To overcome the big size of image 
the best solution proposed is the image compression that is efficient to reduce the global energy consumed in WMSN. The performance of compression techniques [1] depends: image quality; compression ratio; compression speed and consumption power. Figure 2 shows the basic idea of the image compression theory.

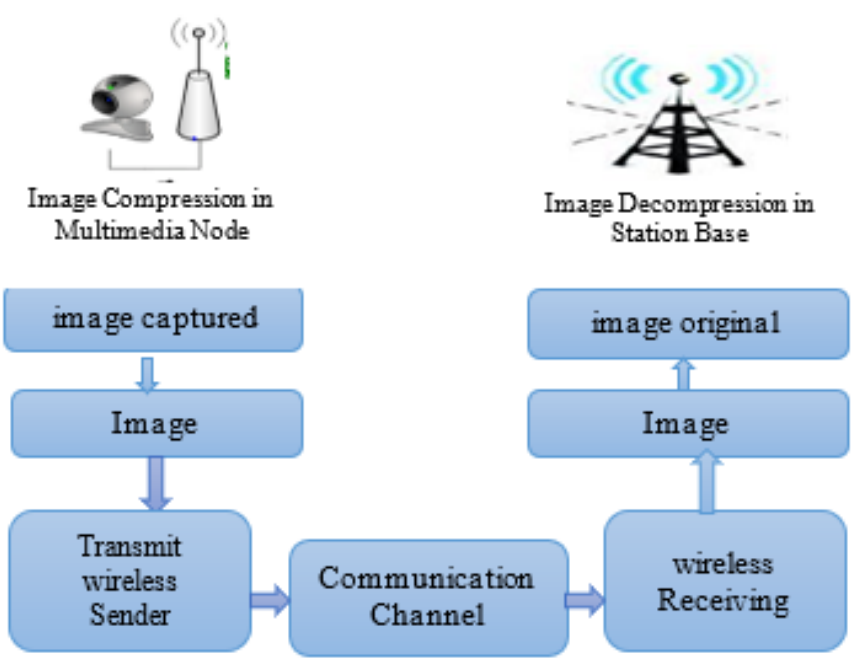

Figure 2. The basic idea of the image compression in WMSN

Previously Many related works have proposed various contributions to improve the compression performance of images in WMSN. In the past few years, Chew et al. [2] reviewed the algorithms of image compression in WMSN. They found that the adequate algorithm is Set Partitioning In Hierarchical Tree (SPIHT) with high simplicity and high compression ratio.

To solve the problem of image compression Cristian Duran [3] presented an evaluation of the performance of five algorithm of images compression on a sensor node. They saw in the results the best algorithm for energy savings in WSN is Sub Sampling (SS).

Nasri et al. [4] suggested images compression scheme using a wavelet image transform and distributed the processing between clusters to minimized the energy and maximized the image quality.

Hussain et al. [5] presented a new method of compression named Image Subtraction with Quantization of image (ISQ). They have shown that ISQ improves the efficiency of energy in each node of the networks.

Mekkaoui et al. [6] demonstrated that the zonal DCT compression method in a WSN reduced the complexity of computational without degradation of the image quality.

Ghorbel et al. [7] determined a practical comparison in the performance between DCT and (DWT) in WMSN. They concluded that DWT is better than DCT. Ghorbel et al. [8] added the parameter of energy consumption (battery lifetime). They found also that DWT is preferable than DCT in quality of image and consumption of energy.

Kumar et al. [9] described a new technique used singular value decomposition (SVD) and block truncation coding (BTC) with (DCT). The results are given by the new method lead to improve the performance of compression better than JPEG.

Ma et al. [10] also discussed three methods of image compression were JPEG2000, EBCOT, and SPIHT. They demonstrated that the preferable method with minimum energy compression and low complexity and high compression ratio is SPIHT.

Kouadria et al. [11] used for image compression in WMSN a technique named Low complexity DCT. The contribution is the compression of the ROI region only from the total image. Simulation results are given a low complexity and good energy compaction.

Benfilali et al. [12] used "background subtraction" technique for the objects detection in system monitoring wireless sensor network.

Aruna Deepthi et al. [13] in their paper two algorithms used the block truncation coding (BTC) and Absolute Moment block truncation coding (AMBTC). The results have shown that AMBTC is speeder than BTC in WSN.

Furthermore, Aruna Deepthi et al. [14] designed in WSNs the different methods of images compression like SVD, NMF, and PNMF with Matlab and RTL schematic. results found that the PNMF was better in energy consumption in-camera nodes.

In Table 1, we have discussed the advantages of various techniques of images compression in WMSN.

Table 1. Advantages of various techniques of images compression in WMSN

\begin{tabular}{|c|c|c|}
\hline Ref & Techniques used & Advantages \\
\hline$[2]$ & $\begin{array}{c}\text { Set Partitioning In } \\
\text { Hierarchical Tree (SPIHT) }\end{array}$ & $\begin{array}{c}\text {-high simplicity } \\
\text { - high compression ratio. }\end{array}$ \\
\hline$[3]$ & $\begin{array}{l}\text { evaluation of the } \\
\text { performance of five } \\
\text { algorithm of images } \\
\text { compression }\end{array}$ & $\begin{array}{l}\text { - Sub Sampling (SS)the } \\
\text { best for energy }\end{array}$ \\
\hline$[4]$ & -wavelet image transform & $\begin{array}{l}\text {-energy minimized } \\
\text {-image quality } \\
\text { maximized }\end{array}$ \\
\hline$[5]$ & $\begin{array}{c}\text { Subtraction with } \\
\text { Quantization of image (ISQ) }\end{array}$ & - improved energy \\
\hline$[6]$ & zonal DCT compression & $\begin{array}{l}\text {-complexity reduced } \\
\text { - image quality. }\end{array}$ \\
\hline $\begin{array}{l}{[7]} \\
{[8]}\end{array}$ & $\begin{array}{c}\text { Comparison between DCT } \\
\text { and (DWT) }\end{array}$ & $\begin{array}{l}\text {-DWT is better than } \\
\text { DCT (image quality, } \\
\text { energy). }\end{array}$ \\
\hline [9] & $\begin{array}{c}\text { Singular Value } \\
\text { Decomposition (SVD) and } \\
\text { Block Truncation coding } \\
\text { (BTC) with (DCT). }\end{array}$ & better than JPEG. \\
\hline$[10]$ & $\begin{array}{l}\text { JPEG2000, EBCOT, and } \\
\text { SPIHT. }\end{array}$ & $\begin{array}{l}\text { SPIHT preferable } \\
\text { (minimum energy, low } \\
\text { complexity, high } \\
\text { compression ratio) }\end{array}$ \\
\hline$[\mathbf{1 1}]$ & $\begin{array}{l}\text { Low complexity DCT of the } \\
\text { ROI region }\end{array}$ & $\begin{array}{l}\text { low complexity and } \\
\text { good energy compaction }\end{array}$ \\
\hline [12] & "background subtraction" & good results \\
\hline$[13]$ & $\begin{array}{l}\text { block truncation coding } \\
\text { (BTC) and Absolute Moment } \\
\text { block truncation coding } \\
\text { (AMBTC). }\end{array}$ & $\begin{array}{l}\text { AMBTC is speeder than } \\
\text { BTC in WSN }\end{array}$ \\
\hline$[14]$ & $\begin{array}{l}\text { SVD, NMF, and PNMF with } \\
\text { Matlab and RTL }\end{array}$ & $\begin{array}{l}\text { PNMF was better in } \\
\text { energy consumption }\end{array}$ \\
\hline
\end{tabular}

All of the cited techniques in the upper are tried to minimize the size of images in the sensor node without degradation of image quality. We can say that the two techniques SIFT and SS preferable than DCT, JPG in terms of energy consumption, and complexity. Usually, in a WMSN the ratio of compression affects the energy of transmission to the base station. Also, the complexity of the technique of compression affected directly the energy of the sensor node. 


\section{FOREST FIRE DETECTION IN WIRELESS MULTIMEDIA SENSOR NETWORK (WMSN)}

Generally forest fires occur due to natural or manmade. In the history of development of systems of fire detection, the use of WSN has been thought of as a key factor for new monitoring system. In this subsection we will provide the different previous related work for the detection of forest fires.

\subsection{Optical sensors and camera surveillance}

These systems are fixed on top of towers with CCD cameras and IR detectors. The main problems for these systems are the expensive cost and the affectation by terrain obstacles, time of day and conditions of weather.

\subsection{Satellite based systems}

For forest fire detection two famous satellites used, the first is advanced very high-resolution radiometer (AVHRR) [15] and the second is Moderate Resolution Imaging Spectroradiometer (MODIS). The drawbacks of this method are the long time and the affectation of weather conditions to the quality of the images [16].

\subsection{Wireless sensor networks}

Several researches have been used of systems based on wireless sensor network like an adequate solution for forest fire detection. In this subsection, we give the popular related works.

In recent years, the researchers attracted to in 2005, Doolin and Sitar [17] suggested to wildfire monitoring the utilization of sensors to sense temperature, humidity, and barometric pressure. The network was more expensive because of the use of GPS device for each sensor node. In the same year, another study [18] used neural WSN and clustering algorithm to realize a real-time forest fire detection system.

In the next year, Son et al. [19] in the south Korean mountains developed a system used to detected forest fire based on WSN where the base station receives temperature, humidity and smoke from sensor nodes. Also, Hurting et al. [20] used WSN for detection of weather status and web cameras to detected fire images.

Garcia et al. [21] at the year 2008 develops a model of forest fire using the data collected by sensors and geographical information of the area.

In the year 2009, Lloret et al. [22] suggested WSN with cameras used internet protocol (IP) to detected forest fire in Spain. Also Conrad et al. [23] realized Forest Fire Detection System with GPS tools in Pennsylvania. In the next year 2010, Aslan [24] described a framework to detected forest fire a using WSN.

Hakilo et al. [25] in 2011, presented a system named wildfire hazard prediction (WFHP) using WSN. WFHP system consisted of 16 sensor that maximized the efficiency and the accuracy of fire detection.

In 2013, Alkhatib [26] used only temperature sensors to implemented WSN model for fire detection. the proposed model offered high precision and cheap cost. Bouabdellah et al. [27] improved the previous study [26] by adding others types of sensors such as humidity, pressure etc. The authors in $[28,29]$ employed the fuzzy logic for intelligent fire detection using different types of sensors.
In the year of 2015 the studies [30, 31] made improvement in fuzzy logic by adding fuzzy logic II, tree classifier, and neighbor-based fuzzy logic algorithms for fire detection.

In the next year 2016 Antonio et al. [32] proposed a WSN hierarchic to early fire detection. Sensor nodes (SNs) collected different data (temperature, humidity, wind speed and direction, $\mathrm{CO}$ and $\mathrm{CO}_{2}$ levels) then send the data collected to SB.

In the year of 2020, Noureddine et al. [33] present an improved edition of the previous system submitted [27] by adding the capabilities of video sensing.

From the above, we can say that in recent years (2005-2020) most of the research on fire monitoring system has used only the scalar sensor like (temperature, humidity, wind speed and direction, $\mathrm{CO}$ and $\mathrm{CO}_{2}$ levels). Our proposed system added the camera sensor to augmented the performance of detection fire. Because when we send image contain fire we can detected more information like size, location and high of fire. Which makes it easier for us to extinguish quickly before it spread.

\section{THE PROTOCOLE OF WSN USED FOR FOREST FIRE DETECTION}

\subsection{Network architecture design fire detection system}

We adopted our protocole proposed by Bouakkaz et al. [34], Bouakkaz and Derdour [35]. Figure 3 gives the basic idea of power efficient grid-chain routing protocol in WSN [35].

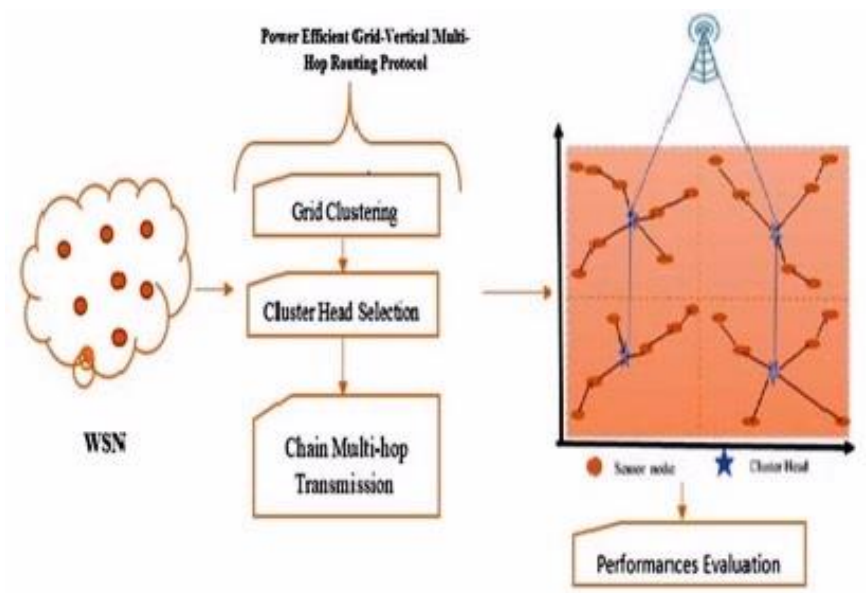

Figure 3. Basic idea of power efficient grid-chain routing protocol in WSN [35]

Our adaptation is the application of proposed protocol for a fire detection system like mentioned in Figure 4 by adding images sensors. So, the data transmitted will be images rather than scalar data. So, we need to use a technique for images compression before the phase of transmission using the same grid multi-hop protocol to reduce the energy consumption of wireless sensor nodes.

We present a suggestion for our forest fire detection monitoring system. We improved the protocol proposed by Bouakkaz et al. [34], Bouakkaz and Derdour [35] by using two type of sensors scalar type for temperature detection and another multimedia type for the fire image detection. 


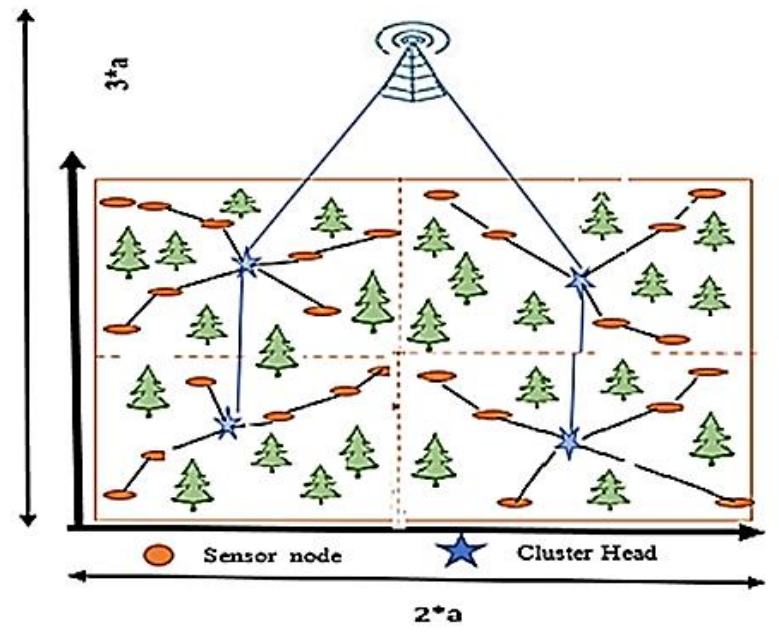

Figure 4. The grid Multi-hop protocol adopted for monitoring forest fire system with WMSN

On the one hand, we select the temperature like the first environmental parameters in our system. We use sensor nodes deployed in various locations of the forest to measure temperature.

On other hand, if the temperature sensors detected a high level of air temperature then the camera sensors deployed in the forest will be activated to capture images to detected if fire exist or no.

\subsection{The data collected levels scheme}

The proposed system is composed of two levels. The first level includes scalar sensors which can sense the temperature degree, the second level contain sensor nodes fitted with cameras which can sense images. The task of detection fire is to find fires in images and cutting the regions containing them and compress images and send it to the sink. The next flow chart Figure 5 explains the two levels of our fire detection system.

\subsection{The image detection and compression scheme used}

At the level of scalar temperature sensor, when a high temperature ( $\mathrm{Ti}>\mathrm{Tth}$ ) was detected, an alarm message will be send to Station Base with the temperature (Ti) and the coordinates $(\mathrm{x}, \mathrm{y})$ of places where fire can be happen. At level of the base of the station, an urgent wake-up message is sent only to the camera sensors in the area (x,y). At the camera sensor level, the following steps will be implemented to detect fires in the images:

- Image capture

- The extraction of items of color $(R, G, B)$

- Define thresholds for each color RGB

- Fire detection maintain only fire part (red color)

- Edge Prewitt detection

- Compress image using Downsample Method

The next scheme Figure 6 explains the different steps in the procedure of images fire detection and compression using Downsample Method in our system.

\subsection{The image compression using Downsample}

Cristian [3] mentioned that the best algorithm for energy savings in WSN is Sub Sampling (SS). For this reason we choose to use this technique after the detection phase of the forest fire the phase compression of images detected will start.

The key idea in image down-sampling is to throw away every other row and column to create new size < orginal size of an image according to the parameter of sampling $\mathrm{K}$.

Table 2 shows an example of image compression Downsample with $\mathrm{k}=2$.

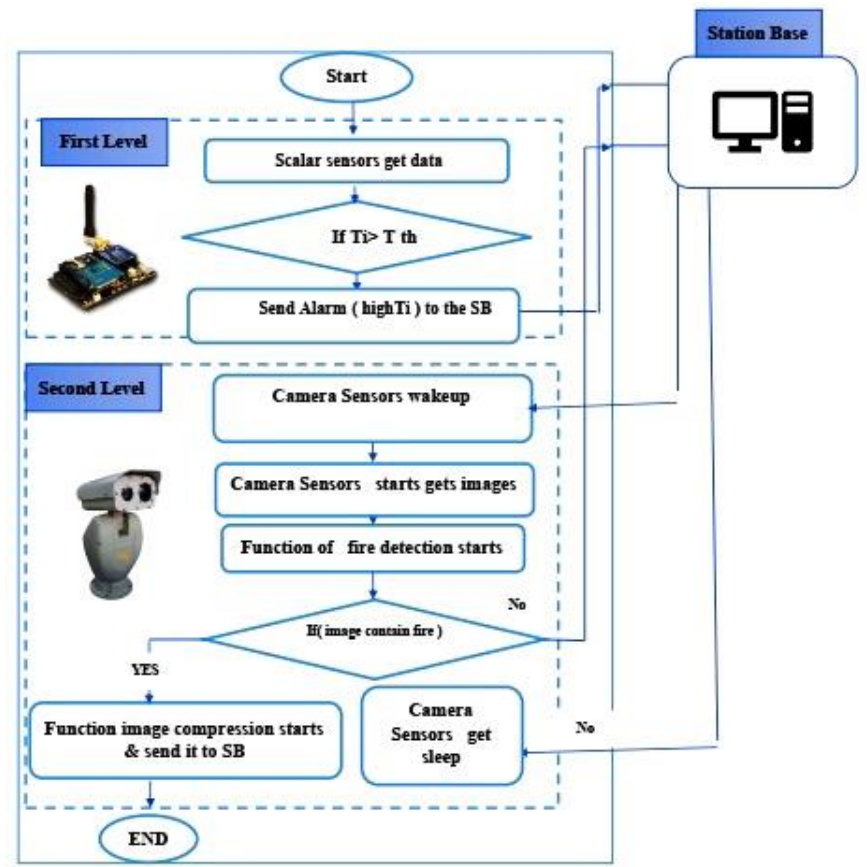

Figure 5. The grid multi-hop protocol adopted for monitoring forest fire system with WMSN

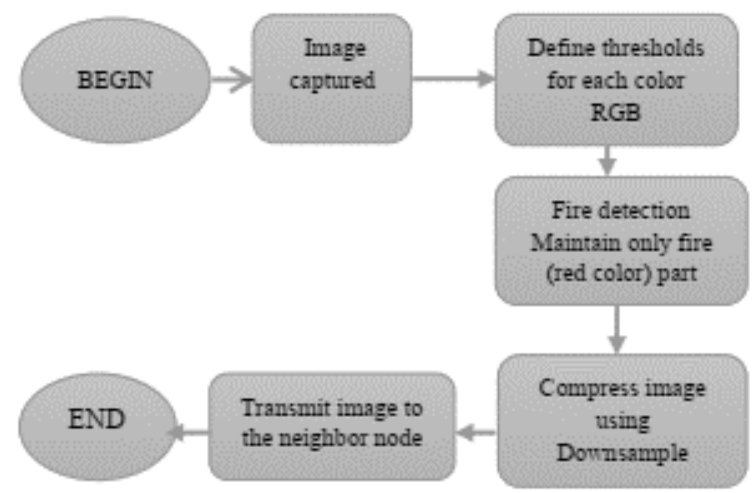

Figure 6. The scheme used to fire detection and compression images

Table 2. An example of image compression Downsample $(\mathrm{k}=2)$

\begin{tabular}{c|c}
\hline $\begin{array}{c}\text { Image original } \\
{[\mathrm{M}=6 ; \mathrm{N}=4]}\end{array}$ & Downsample an image by $\mathrm{k}=\mathbf{2}$ \\
\hline & \\
\hline & \\
\hline
\end{tabular}

\section{SIMULATION AND RESULTS}

Simulation is achieved by MATLAB 9, the first column in 
the Table 3 shows some images captured contain forest fire, the second column include the original size of each image where the third column contain each image with only part of forest fire detected using our approach proposed. The fourth column include each image with Downsample method of compression used, finally the fifth column contain the size after compression.

We demonstrated by simulation that the using of two type of sensors with grid multi-hop protocol enhances the energy efficiency especially node camera that will be activated only when the temperature ( $\mathrm{Ti}>\mathrm{T}$ threshold).

For the compression of images, we used the Downsample method because this technique is very simple and have high rate compression also it is easy to implement, and have not need big processing power as other methods.

Performance rating has indicated that the node equipped camera consume less energy than $50 \%$ after compression that lead to maximizing the life time of the network.

Table 3. Simulation results

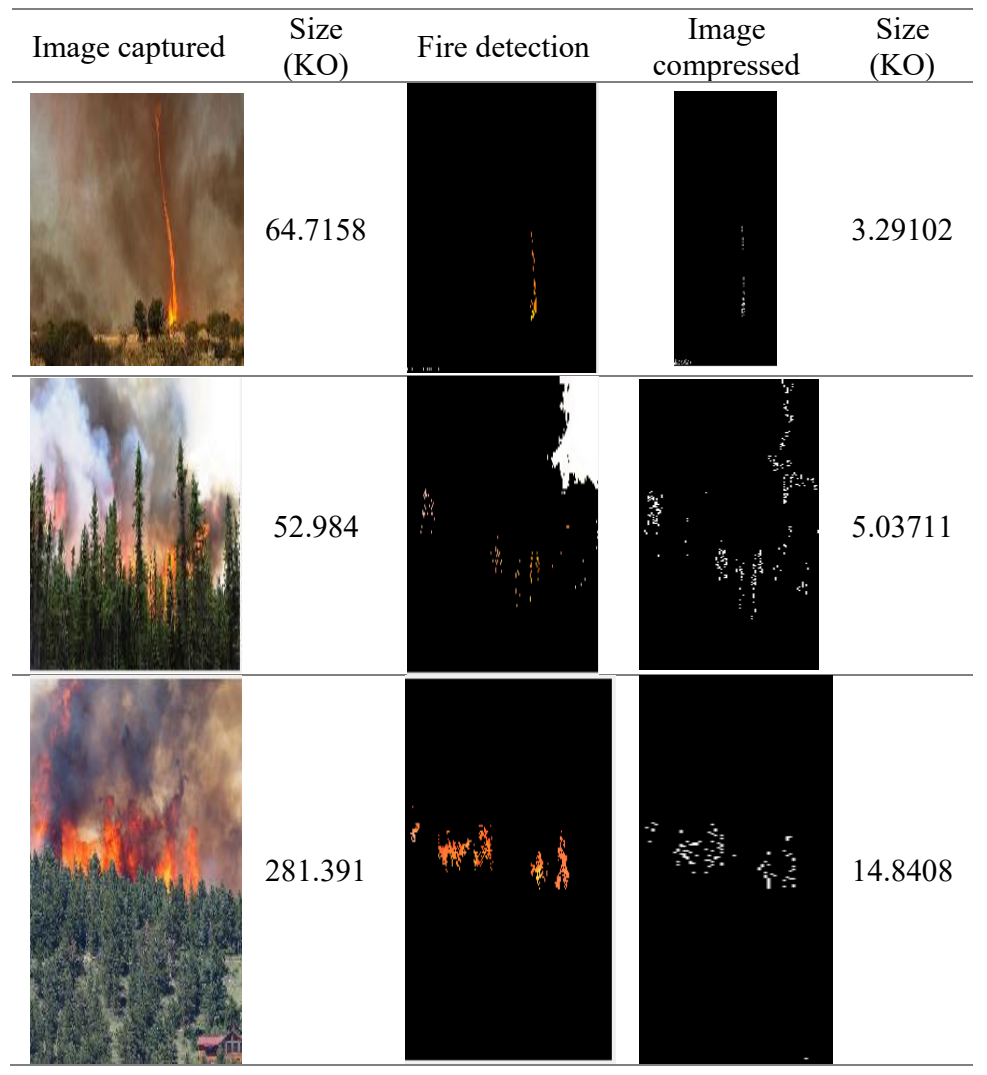

\section{CONCLUSION}

Forest fire is one of the most catastrophes frequently happen in all the world. The goal of this paper article was combined between two technologies, wireless multimedia sensor networks and image compression, to detect forest fire incidents. When we use image sensor we can detected more information like size, location and high of fire. Which makes it easier for us to extinguish quickly before it spread.

We have suggested new a protocol with two types of sensors (scalar and multimedia) for forest fire detection system based on WMSN.

Our technique suggested is critical solution for the future of forest fire detection as demonstrated by the obtained results. In our future work, we try to implement our protocol proposed in a forest in Algeria. We also can add other types of sensors such as air humidity sensors and smoke sensors.

\section{REFERENCES}

[1] Samra, H.S. (2012). Image compression techniques. International Journal of Computers \& Technology, 2(2): 49-52.
[2] Chew, L.W., Ang, L.M., Seng, K.P. (2008). Survey of image compression algorithms in wireless sensor networks. 2008 International Symposium on Information Technology, Kuala Lumpur, Malaysia, pp. 1-9. https://doi.org/10.1109/ITSIM.2008.4631875

[3] Cristian, D.F. (2009). Transmission of images on wireless sensor networks under the constraint of energy. Ph.D. Thesis, Henri Poincary University, Center of Research in Automation of Nancy.

[4] Nasri, M., Helali, A., Sghaier, H., Maaref, H. (2011). Adaptive image compression technique for wireless sensor networks. Computers \& Electrical Engineering, 37(5): https://doi.org/10.1016/j.compeleceng.2011.08.001

[5] Hussain, S.A., Razzak, M.I., Minhas, A.A., Sher, M., Tahir, G.R. (2010). Energy efficient image compression in wireless sensor networks. 2010, International Journal on Signal and Image Processing, 1(2).

[6] Makkaoui, L., Lecuire, V., Moureaux, J.M. (2010). Fast zonal DCT-based image compression for Wireless Camera Sensor Networks. 2010 2nd International Conference on Image Processing Theory, Tools and Applications. https://doi.org/10.1109/ipta.2010.5586798

[7] Ghorbel, O., Jabri, I., Ayedi, W. (2011). Experimental 
study of compressed images transmissions through WSN. ICM 2011 Proceeding, Hammamet, Tunisia, pp. 1-6. https://doi.org/10.1109/ICM.2011.6177378

[8] Ghorbel, O., Ayedi, W., Jmal, M.W., Abid, M. (2011). Image compression in WSN: performance analysis. 2012 IEEE 14th International Conference on Communication Technology, Chengdu, China. https://doi.org/10.1109/ICCT.2012.6511432

[9] Kumar, V., Kumar, A., Bhardwaj, A. (2012). Performance evaluation for image compression techniques. 2012 International Conference on Devices, Circuits and Systems (ICDCS), Coimbatore, India, pp. 447-450.

https://doi.org/10.1109/ICDCSyst.2012.6188797

[10] Ma, T., Hempel, M., Peng, D.M., Sharif, H. (2013). A survey of energy-efficient compression and communication techniques for multimedia in resource constrained systems. IEEE Communications Surveys \& Tutorials, 15(3): 963-972. https://doi.org/10.1109/SURV.2012.060912.00149

[11] Kouadria, N., Doghmane, N., Messadeg, D., Harize, S. (2013). Low complexity DCT for image compression in wireless visual sensor networks. Electronics Letters, 49(24): 1531-1532. https://doi.org/10.1049/el.2013.2781

[12] Benfilali, M., Abdkader, G., Sofiane, B.H. (2016). Recent image compression strategies to reduce the cost of transmission in wireless sensor networks. International Journal of RFID Security and Cryptography (IJRFIDSC), 4(1): 177-184.

[13] Aruna Deepthi, S., Sreenivasa Rao, E., Giri Prasad, M.N. (2017). Image compression for wireless sensor networks. 2017 IEEE International Conference on Smart Technologies and Management for Computing, Communication, Controls, Energy and Materials (ICSTM), Chennai, India. https://doi.org/10.1109/ICSTM.2017.8089170

[14] Aruna Deepthi, S., Sreenivasa Rao, E., Giri Prasad, M.N. (2019). RTL implementation of image compression techniques in WSN. International Journal of Electrical and Computer Engineering (IJECE), 9(3): 1750-1756. http://doi.org/10.11591/ijece.v9i3.pp1750-1756

[15] Flannigan, M.D., Haar, T.H.V. (1986). Forest fire monitoring using NOAA satellite AVHRR. Canadian Journal of Forest Research, 16(5): 975-982. https://doi.org/10.1139/x86-171

[16] Justice, C., Townshend, J. R., Vermote, E., Masuoka, E., Wolfe, R., Saleous, N., Roy, D.P., Morisette, J. (2002). An overview of MODIS Land data processing and product status. Remote Sensing of Environment, 83(1-2): 3-15. https://doi.org/10.1016/s0034-4257(02)00084-6

[17] Doolin, D., Sitar, N. (2005). Wireless sensors for wild fire monitoring. Proceedings of SPIE - The International Society for Optical Engineering.

[18] Yu, L.Y., Wang, N., Meng, X.Q. (2005). Real-time forest fire detection with wireless sensor networks. In Proceedings of the International Conference on Wireless Communications, Networking and Mobile Computing (WCNM $\left.{ }^{\text {ee }} 05\right), \quad$ pp. 1214-1217. https://doi.org/10.1109/WCNM.2005.1544272

[19] Son, Y., Her, Y. (2006). A design and implementation of forest-fires surveillance system based on wireless sensor networks for south Korea mountains. International Journal of Computer Science and Network Security, 6(9): 124-130.
[20] Hartung, C., Han, R., Seielstad, C., Holbrook, S. (2006). FireWxNet: Amulti-tiered portable wireless system for monitoring weather conditions in wildland fire environments. Proceedings of the 4th International Conference on Mobile Systems, Applications and Services (MobiSys "e06), ACM, Uppsala, Sweden, pp. 28-41. https://doi.org/10.1145/1134680.1134685

[21] Garcia, E.M., Serna, M.A., Bermudez, A., Casado, R. (2008). Simulating a WSN-based wildfire fighting support system. Proceedings of the International Symposium on Parallel and Distributed Processing with Applications (ISPA ${ }^{\text {ee }}$ 08), pp. 896-902. https://doi.org/10.1109/ISPA.2008.23

[22] Lloret, J., Garcia, M., Bri, D., Sendra, S. (2009). A wireless sensor network deployment for rural and forest fire detection and verification. Sensors, 9(11): 8722-8747. https://doi.org/10.3390/s91108722

[23] Conrad, A., Liu, Q., Russell, J., Lalla, J. (2009). Enhanced Forest Fire Detection System with GPS Pennsylvania.

[24] Aslan, Y. (2010). A framework for the use of wireless sensor networks in the forest fire detection and monitoring. Department of Computer Engineering, The Institute of Engineering and Science Bilkent University.

[25] Sabit, H., Al-Anbuky, A., GholamHosseini, H. (2011). Wireless sensor network based wildfire hazard prediction system modeling. Procedia Computer Science, 5: 106114. https://doi.org/10.1016/j.procs.2011.07.016

[26] Alkhatib, A.A. (2013). Smart and low cost technique for forest fire detection using wireless sensor network. International Journal of Computer Applications, 81(11): 12-18. https://doi.org/10.5120/14055-2044

[27] Bouabdellah, K., Noureddine, H., Larbi, S. (2013). Using wireless sensor networks for reliable forest fires detection. Procedia Computer Science, 19: 794-801. https://doi.org/10.1016/j.procs.2013.06.104

[28] Bolourchi, P., Uysal, S. (2013). Forest fire detection in wireless sensor network using fuzzy logic. In: IEEE 2013 Fifth International Conference on Computational Intelligence, Communication Systems and Networks (CICSyN), Madrid, Spain, pp. 83-87. https://doi.org/10.1109/CICSYN.2013.32

[29] Ammar, M.B., Souissi, R. (2014). A new approach based on wireless sensor network and fuzzy logic for forest fire detection. International Journal of Computer Applications, $\quad$ 89(2): $48-55$. https://doi.org/10.5120/15477-4169

[30] Maksimović, M., Vujović, V., Perišić, B., Milošević, V. (2015). Developing a fuzzy logic based system for monitoring and early detection of residential fire based on thermistor sensors. Computer Science and Information $\quad$ Systems, $12(1)$ : 63-89. https://doi.org/10.2298/csis140330090m

[31] Wang, B., Zhuang, A., Sun, H., Li, T., Sun, X. (2015). An improved spatial-based fuzzy logic event detecting algorithm for wireless sensor networks. International Journal of u- and e-Service, Science and Technology, 8(4): https://doi.org/10.14257/ijunesst.2015.8.4.24

[32] Molina-Pico, A., Cuesta-Frau, D., Araujo, A., Alejandre, J., Rozas, A. (2016). Forest monitoring and wildland early fire detection by a hierarchical wireless sensor network. Journal of Sensors, 2016: 1-8. https://doi.org/10.1155/2016/8325845 
[33] Noureddine, H., Bouabdellah, K. (2020). Using wireless multimedia sensor networks to enhance early forest fire detection. International Journal of Distributed Systems and Technologies, 11(3): $1-21$ https://doi.org/10.4018/IJDST.2020070101

[34] Bouakkaz, F., Makhlouf, D., Wided, A. (2020). Improved vitality of wireless sensor network using grid clustering with multi-hop transmission protocol routing. Telecommunications and Radio Engineering, 79(6): 521532. https://doi.org/10.1615/TelecomRadEng.v79.i6.60

[35] Bouakkaz, F., Derdour, M. (2020). Maximizing WSN life using power efficient grid-chain routing protocol (PEGCP). Wireless Personal Communications. https://doi.org/10.1007/s11277-020-07908-9 